\title{
Impact of Different Mass Drug Administration Strategies for Gaining and Sustaining Control of Schistosoma mansoni and Schistosoma haematobium Infection in Africa
}

Charles H. King, ${ }^{1,2 \star}$ Nupur Kittur, ${ }^{2}$ Sue Binder, ${ }^{2}$ Carl H. Campbell Jr., ${ }^{2}$ Eliézer K. N'Goran, ${ }^{3,4}$ Aboulaye Meite, ${ }^{5}$ Jürg Utzinger, ${ }^{6,7}$ Annette Olsen, ${ }^{8}$ Pascal Magnussen, ${ }^{9}$ Safari Kinung'hi, ${ }^{10}$ Alan Fenwick, ${ }^{11}$ Anna E. Phillips, ${ }^{11}$ Pedro H. Gazzinelli-Guimaraes ${ }^{11}$ Neerav Dhanani, ${ }^{11}$ Josefo Ferro, ${ }^{12}$ Diana M. S. Karanja, ${ }^{13}$ Pauline N. M. Mwinzi, ${ }^{13}$ Susan P. Montgomery, ${ }^{14}$ Ryan E. Wiegand, ${ }^{6,7,14}$ William Evan Secor, ${ }^{14}$ Amina A. Hamidou, ${ }^{15}$ Amadou Garba, ${ }^{16}$ and Daniel G. Colley ${ }^{2,17}$

${ }^{1}$ Center for Global Health and Diseases, Case Western Reserve University, Cleveland, Ohio; ${ }^{2}$ Schistosomiasis Consortium for Operational Research and Evaluation, Center for Tropical and Emerging Global Diseases, University of Georgia, Athens, Georgia; ${ }^{3}$ Unité de Formation et de Recherche Biosciences, Université Félix Houphouët-Boigny, Abidjan, Côte d'Ivoire; ${ }^{4}$ Centre Suisse de Recherches Scientifiques en Côte d'Ivoire, Abidjan, Côte d'Ivoire; ${ }^{5}$ Programme National de Lutte Contre les Maladies Tropicales Négligées à Chimiothérapie Préventive (PNLMTN-CP), Abidjan, Côte d'Ivoire; ' 5 wiss Tropical and Public Health Institute, Basel, Switzerland; ${ }^{7}$ University of Basel, Basel, Switzerland; ${ }^{8}$ Section for Parasitology and Aquatic Pathobiology, Faculty of Health and Medical Sciences, University of Copenhagen, Copenhagen, Denmark; ${ }^{9}$ Centre for Medical Parasitology, Faculty of Health and Medical Sciences, University of Copenhagen, Copenhagen, Denmark; ${ }^{10}$ National Institute for Medical Research, Mwanza, Tanzania; ${ }^{11}$ Schistosomiasis Control Initiative, Imperial College, London, United Kingdom; ${ }^{12}$ Catholic University of Mozambique, Beira, Mozambique; ${ }^{13}$ Centre for Global Health Research, Kenya Medical Research Institute, Kisumu, Kenya; ${ }^{14}$ Centers for Disease Control and Prevention, Atlanta, Georgia; ${ }^{15}$ Réseau International Schistosomoses, Environnement, Aménagement et Lutte (RISEAL-Niger), Niamey, Niger; ${ }^{16}$ Department of Control of Neglected Tropical Diseases, Preventive Chemotherapy and Transmission Control Unit, World Health Organization, Geneva, Switzerland; ${ }^{17}$ Department of Microbiology, University of Georgia, Athens, Georgia

\begin{abstract}
This report summarizes the design and outcomes of randomized controlled operational research trials performed by the Bill \& Melinda Gates Foundation-funded Schistosomiasis Consortium for Operational Research and Evaluation (SCORE) from 2009 to 2019. Their goal was to define the effectiveness and test the limitations of current WHOrecommended schistosomiasis control protocols by performing large-scale pragmatic trials to compare the impact of different schedules and coverage regimens of praziquantel mass drug administration (MDA). Although there were limitations to study designs and performance, analysis of their primary outcomes confirmed that all tested regimens of praziquantel MDA significantly reduced local Schistosoma infection prevalence and intensity among school-age children. Secondary analysis suggested that outcomes in locations receiving four annual rounds of MDA were better than those in communities that had treatment holiday years, in which no praziquantel MDA was given. Statistical significance of differences was obscured by a wider-than-expected variation in community-level responses to MDA, defining a persistent hot spot obstacle to MDA success. No MDA schedule led to elimination of infection, even in those communities that started at low prevalence of infection, and it is likely that programs aiming for elimination of transmission will need to add supplemental interventions (e.g., snail control, improvement in water, sanitation and hygiene, and behavior change interventions) to achieve that next stage of control. Recommendations for future implementation research, including exploration of the value of earlier program impact assessment combined with intensification of intervention in hot spot locations, are discussed.
\end{abstract}

\section{INTRODUCTION}

In 2009, the Bill \& Melinda Gates Foundation (BMGF) funded the Schistosomiasis Consortium for Operational Research and Evaluation (SCORE), based at the University of Georgia. The seven most extensive projects at the core of SCORE's operational research agenda involved large-scale, randomized comparison trials of different approaches to mass drug administration (MDA) using praziquantel for control of either Schistosoma mansoni or of Schistosoma haematobium in endemic areas of sub-Saharan Africa. ${ }^{1}$ In aiming to provide program managers and policy makers with better evidence for decision-making, SCORE's research objective for these studies was to generate data on the relative effectiveness of community-wide treatment (CWT) versus school-based treatment $(\mathrm{SBT})^{2,3}$ and on the relative effectiveness of annual praziquantel drug delivery as compared with schedules that involve holiday years-that is, years without praziquantel MDA. ${ }^{4-8}$ The focus on sub-Saharan Africa was based on this region having the greatest burden of schistosomiasis globally

*Address correspondence to Charles H. King, Center for Global Health and Diseases, CWRU School of Medicine, 2109 Adelbert Rd., Cleveland, $\mathrm{OH}$ 44106. E-mail: chk@cwru.edu and the greatest need for information about optimal implementations at national and subnational scales.

After extensive consultation in 2009 with program managers, field trial researchers, biostatisticians, and representatives of the WHO, SCORE designed multiyear, large-scale cluster-randomized trials to study options for program implementation in areas having $\geq 10 \%$ Schistosoma infection prevalence among school-age children (SAC). ${ }^{1}$ At the time, WHO guidelines recommended every-other-year praziquantel treatment of all SAC in communities having SAC prevalence between $10 \%$ and $49 \%$, and annual SAC treatment for communities having SAC prevalence $\geq 50 \% .{ }^{9}$ For both categories, concurrent treatment of high-risk adults (i.e., those having regular contact with infested water, such as fishermen, farmers, irrigation workers, or women regularly coming in contact as part of their domestic tasks) was recommended, with the added proviso that in the areas of highest prevalence $(\geq 50 \%)$, the entire community might need to be treated. ${ }^{9}$

In 2009, SCORE stakeholder partner deliberations agreed that these existing thresholds for implementation of mass treatment needed refinement and a better evidence base to establish the relative benefits of different age-group coverage formats and different schedules of targeted mass praziquantel delivery. ${ }^{1}$ In particular, it seemed quite unlikely that communities starting at a prevalence of $10 \%$ Schistosoma infection 
would have the same response to mass treatment as communities starting at a several fold higher prevalence (e.g., $49 \%$ ), although they were classified in the same risk group by WHO guidelines. ${ }^{9}$ There was interest in finding out whether, when compared with SBT, using one or more years of CWT would have a greater impact on the prevalence and intensity of infection if, for example, CWT could achieve better coverage of SAC who did not attend school ${ }^{2}$ or better coverage of highrisk adults. There also was a lack of data as to whether everyother-year delivery of MDA could be "good enough" for morbidity control, ${ }^{10}$ that is, near elimination of heavy infections (defined as $\geq 400 \mathrm{~S}$. mansoni eggs per gram of stool or $\geq 50 \mathrm{~S}$. haematobium eggs per $10 \mathrm{~mL}$ of urine). ${ }^{9}$

As a result, two types of SCORE treatment trials were developed to focus separately on communities having starting prevalence $10-24 \%$, designated as sustaining control studies, and on communities having starting prevalence $\geq 25 \%$, designated as gaining control studies. Sustaining control studies in S. mansoni areas were conducted in Côte d'Ivoire and in western Kenya. Gaining control studies in S. mansoni areas were performed in Kenya and in Tanzania, and a gaining control study was performed in Mozambique in an area endemic for $S$. haematobium. ${ }^{1}$ Niger began both gaining and sustaining control studies in areas endemic for $S$. haematobium. However, these studies had then to be modified, as detailed in the following paragraphs.

This article summarizes the results of the SCORE gaining and sustaining studies and the redesigned Niger study. A separate report in this issue ${ }^{11}$ presents the results of separate SCORE studies that focused on "moving toward elimination" on Zanzibar and in S. haematobium-endemic areas of northern and central Côte d'Ivoire. ${ }^{12}$

\section{SCHISTOSOMIASIS CONSORTIUM FOR OPERATIONAL RESEARCH AND EVALUATION STUDY DESIGNS}

Development of harmonized protocols for the SCORE gaining and sustaining control studies has been described in detail in a previous publication. ${ }^{1}$ Briefly, it was decided that sustaining control studies (in locations with starting Schistosoma prevalence of 10-24\%) would have communitylevel randomization to one of three different arms that compared different schedules of SBT with praziquantel. The higher prevalence gaining control areas were believed to need more aggressive intervention, but there was considerable uncertainty (and therefore equipoise) about whether more aggressive population coverage or more frequent drug delivery would yield better outcomes in terms of reducing prevalence and intensity of Schistosoma infection. ${ }^{1}$ Therefore, communities in the gaining control studies (starting prevalence $\geq 25 \%$ ) were randomized to one of six arms: the three SBT schedules used in the sustaining control studies, plus three additional arms that included CWT. A diagram of the treatments given in the different study arms of the gaining and sustaining control studies is presented in Figure 1.

The studies were unblinded, community-level, clusterrandomized control trials, with communities receiving either CWT, SBT, or a CWT to SBT transition, with or without praziquantel holidays during the 4 years of trial. Although all school-age children were targeted for MDA, the chosen study endpoints were based on the evaluation of infection prevalence and intensity outcomes among 9-12-year-old children, the highest risk group, in each community in Year 5. Community eligibility was determined by initial preenrollment screening surveys for $S$. mansoni or $S$. haematobium infection among fifty 13-14-year-old schoolchildren. This screening age range was chosen for the community eligibility assessment because, ethically, it was felt that any child diagnosed as infected with schistosomes deserved prompt individual treatment and that pre-study treatment of a small number of 13-14-year-old individuals would not influence the underlying community baseline status among the trial's sentinel 9-12year-old age-group. In the screening phase, S. mansoni infection was detected by duplicate Kato-Katz thick smears ${ }^{13}$ taken from a single daily stool and $S$. haematobium infection was diagnosed by the detection of microhematuria by urine reagent strip testing. ${ }^{14,15}$

Sustaining control studies. These cluster-randomized studies involved comparison of three different regimens of SBT of SAC in medium prevalence communities. The harmonized criteria for SCORE studies on sustaining control (see protocol in Supplemental Appendix A for details) were as follows:

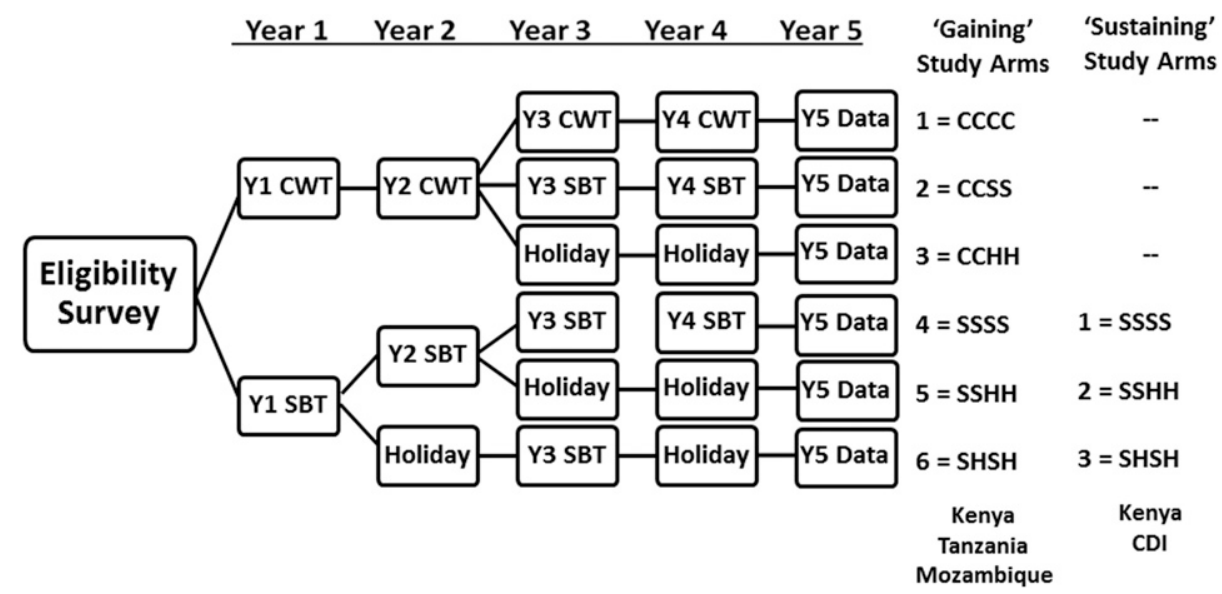

FIGURE 1. Study arms and timeline for the Schistosomiasis Consortium for Operational Research and Evaluation studies of gaining and sustaining control of schistosomiasis in sub-Saharan Africa. $\mathrm{CDI}=\mathrm{Côte}$ d'Ivoire; $\mathrm{CWT}$ or $\mathrm{C}=$ community-wide treatment; $\mathrm{H}=\mathrm{drug}$ holiday-ayear when no praziquantel mass drug administration was provided; SBT or $\mathrm{S}=$ school-based treatment. 


\section{Community inclusion criteria}

- Schistosoma infection prevalence of $10-24 \%$ (by egg detection) among children aged 13-14 years in eligibility surveys before the start of the study, or, for $S$. haematobium studies, a 5-20\% prevalence of microhematuria by reagent strip testing. The option for microhematuria-based surveys for village eligibility was based on their relative ease of performance and the rapidity of their results. The lower 5-20\% cutoffs for $S$. haematobium prevalence estimation by dipstick testing (versus egg count prevalence) were chosen based on the past experience of SCORE investigators working in Kenya, Zanzibar, and Niger, who had established that only about $50-60 \%$ of light and very light infections found by egg filtration have detectable dipstick hematuria. ${ }^{16,17}$ For the preliminary village eligibility surveys, but not the SCORE studies themselves, 5-20\% dipstickpositive prevalence among 13-14 year olds was taken to indicate a moderate prevalence community, where S. haematobium egg count prevalence was likely to be in the $10-24 \%$ range.

- Presence of a community primary school with at least 100 children aged 9-12 years who were available for surveillance. Adjacent communities could be combined as an implementation unit to make up this number of children to treat and monitor.

- Community-level assent, and willingness of parents/ guardians to provide written informed consent for individual children's participation. Children's assent for testing and praziquantel treatment.

2. Community exclusion criteria

- Communities having baseline prevalence outside the $10-24 \%$ range.

- High rates (>10\%) of mixed S. mansoni and S. haematobium infection.

3. The primary outcomes of the study were the prevalence and intensity of infection among a random sample of 100 local SAC, aged 9-12 years in Year 5, after 4 years of implementation of the community's assigned treatment schedule.

4. As shown in Figure 1, the three study arms for comparison were as follows: Arm 1, annual SBT for 4 years; Arm 2, annual SBT for 2 years, followed by 2 years without praziquantel treatment (praziquantel holiday years); and Arm 3, two rounds of SBT given every other year, starting in Year 1 , with praziquantel holidays in the intervening years (years 2 and 4).

5. Primary statistical analysis focused on comparing the WHO-recommended intervention, that is, every-otheryear MDA, ${ }^{9,18}$ to more aggressive annual treatment, or to the impact of two annual treatments followed by a 2-year pause.

6. Data collected for secondary analyses were focused on community-level factors related to treatment response and the relative costs involved in implementing each study arm. These factors included information on local water and sanitation features, interval rainfall and drought, and the treatment coverage achieved among SAC in each community. In addition to monitoring 9-12-year-old children, the prevalence and intensity of infection among 100 firstyear schoolchildren (aged 5-8 years) was assessed in the first and fifth year of each study as an indirect gage of recent rates of local transmission.
Gaining control studies. These cluster-randomized studies involved comparison of six different regimens of CWT and/or SBT in communities with baseline infection prevalence $\geq 25 \%$. The harmonized criteria for SCORE studies on gaining control were as follows:

\section{Community inclusion criteria}

- Schistosoma infection prevalence of $\geq 25 \%$ (by egg detection) among children aged 13-14 years in eligibility surveys performed before the start of the study, or, for $S$. haematobium studies, $a \geq 21 \%$ prevalence of microhematuria on reagent strip testing.

- School size and participation criteria as for sustaining control studies, described earlier.

2. Community exclusion criteria

- Communities having baseline prevalence below $25 \%$

- High rates (> 10\%) of mixed S. mansoni and S. haematobium infection.

3. As in the sustaining control studies, the primary study outcomes were the prevalence and intensity of infection among a random sample of local 9-12-year-old children in Year 5, after 4 years of implementation of the community's assigned treatment schedule.

4. As shown in Figure 1, the six study arms included in gaining control studies were as follows: Arm 1, CWT annually for 4 years; Arm 2, CWT annually for 2 years followed by SBT for 2 years; Arm 3, CWT for 2 years followed by two praziquantel holiday years; and Arms 4-6 were identical to Arms 1-3 of the sustaining control studies, described earlier.

5. Primary statistical analysis focused on comparing the WHO standard of care (SBT every year, given when baseline SAC prevalence is $\geq 50 \%$ ) or every-other-year MDA (when baseline prevalence is $10-49 \%)^{9,18}$ to more aggressive CWT for 2 or 4 years, or to CWT tapering to SBT over 4 years.

6. As was performed for the sustaining control studies, data collected for secondary analyses included assessment of community-level factors related to treatment response, treatment coverage, and the relative costs involved in implementing each study arm. The prevalence and intensity of infection were also assessed in 100 previously untreated 5-8-year-old first-year students in each community as an indicator of ongoing transmission. For these gaining control studies, 50 adults aged 20-55 years in each community were also examined in years 1 and 5 both to gage the influence of baseline adult infection status on local response to treatment intervention and assess the direct or indirect impact of 4 years of local MDA implementation on the adult levels of infection in the final year (Year 5).

Power analysis performed during development of the harmonized protocols indicated that the inclusion of 25 communities in each study arm, with monitoring of 100 children per community, would have $90 \%$ power to detect a significant (two-tailed $\alpha=0.05$ ) between-arm difference of $11 \%$ and $9 \%$ in the Year 5 outcomes for gaining and sustaining studies, respectively. The assumed overdispersion factor was $\phi=5.0$, and the expected prevalence shift was from an average $50 \%$ prevalence in Year 1, to $15 \%$ prevalence in Year 5 in gaining control studies and from $24 \%$ to $10 \%$ in sustaining control studies. ${ }^{1}$ The final selection of 25 communities per arm per study was based on 
these projections and also the practical limitations (program capacity for implementation) of proposed partner sites in endemic countries.

Additional operational research on morbidity control was layered onto these gaining control studies. Cohort morbidity studies on the impact of treatment on early childhood morbidity were embedded in 8-12 communities in each of the S. haematobium and S. mansoni gaining control studies. ${ }^{10}$ Their results are described in further detail in a separate article in this issue. ${ }^{19} \mathrm{~A}$ subset of communities in the gaining control studies were also used for the companion studies of intermediate host snail abundance and infection ${ }^{20}$ and the influence of MDA on population genetics of the respective Schistosoma parasites. ${ }^{21}$

Midpoint structural reorganization of the Niger gaining and sustaining control studies. In the gaining and sustaining control studies in Niger, there was a significant protocol deviation with respect to randomization of study communities, in which geographically clustered groups of 25 communities were randomly assigned to each of the study arms rather than having the different community interventions distributed randomly.

Subsequently, the Niger protocols were modified after Year 2. The new objective was to compare twice-yearly treatment to once-yearly treatment. In Year 3, all study arms for both studies were divided into two parts, with half of the communities in each category randomized to receive subsequent twice-a-year (biannual) MDA, whereas the other half received annual MDA (see Figure 2). This created three distinct randomized comparison sub-studies. Sub-study $A$ was derived from communities initially enrolled in the Niger sustaining control study, and sub-studies $B$ and $C$ were derived from communities initially enrolled in the gaining control study. The sub-studies A, B, and C were analyzed separately.

1. Primary outcomes of the study were the prevalence and intensity of Schistosoma infection among a random sample of local SAC, aged 9-12 years, in Year 5, after 4 years of implementation of the community's assigned treatment schedule.

2. Data collected for secondary analysis was the same as for the other studies of gaining and sustaining control. As in those studies, the prevalence and intensity of infection was additionally assessed in 100 first-year students in years 1 and 5 . In those communities that were initially part of the gaining control study, data were also collected on 50 adults aged $20-55$ years in years 1 and 5 .

\section{KEY FINDINGS FROM THE GAINING AND SUSTAINING CONTROL STUDIES}

In aggregate, the large-scale SCORE gaining and sustaining studies provided important new evidence on programmatically critical issues relating to how best to gain and sustain the morbidity control of schistosomiasis. Mass drug administration with praziquantel reduced community-level prevalence and the mean intensity of Schistosoma infection across all study arms and in all SCORE study countries (AE Phillips, et al., personal communication and M Ouattara et al., personal communication). ${ }^{22-28}$ Figure 3 shows the Year 1 (baseline) and Year 5 prevalence values by treatment study arm for the sustaining control studies for S. mansoni in Côte d'Ivoire and Kenya, the gaining control studies for S. mansoni in Kenya and Tanzania, and the gaining control study for $S$. haematobium in Mozambique. Prevalence by intensity of

\begin{tabular}{lllll} 
Year 1 & Year $\mathbf{2}$ & Year 3 & Year 4 & Year 5 \\
\hline
\end{tabular}

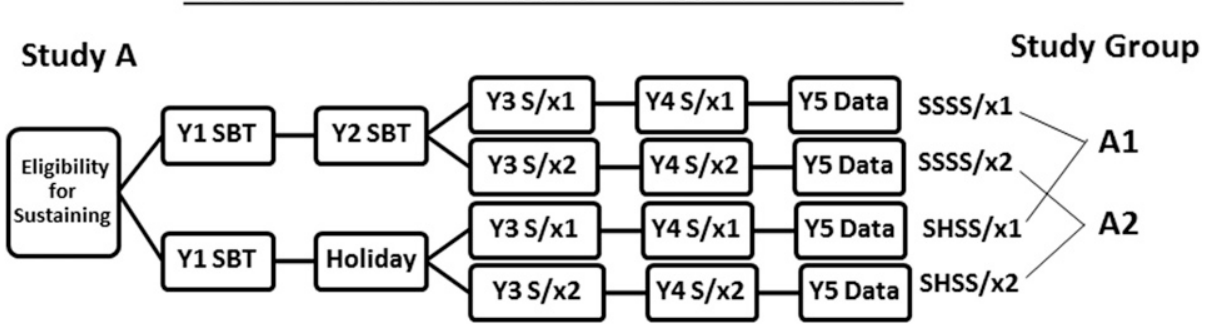

Study B

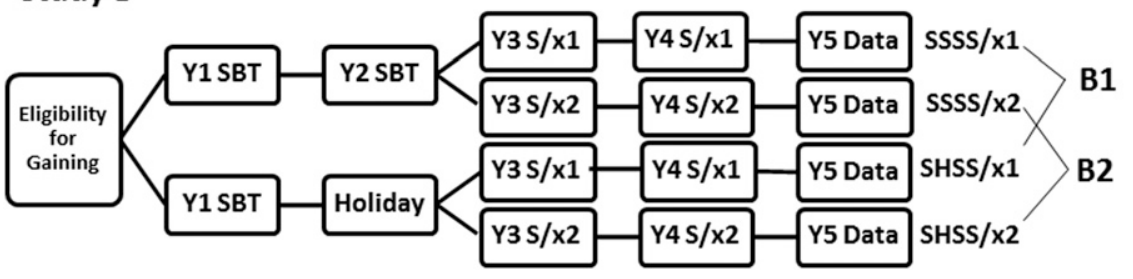

Study C

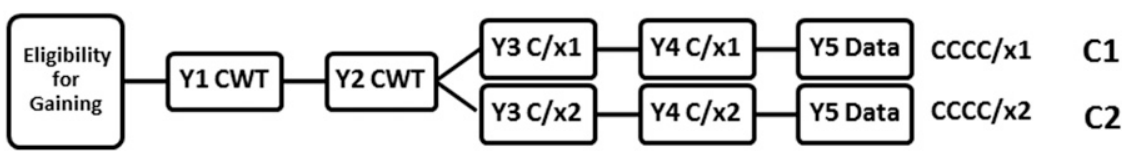

FIGURE 2. Revised study design for Schistosomiasis Consortium for Operational Research and Evaluation's Niger Schistosoma haematobium control studies. CWT or $\mathrm{C}=$ community-wide treatment; $\mathrm{H}=$ drug holiday-a year when no praziquantel mass drug administration (MDA) was provided; SBT or $S=$ school-based treatment; $\mathrm{x} 1=$ one MDA per year in years 3 and $4 ; \mathrm{x} 2=$ two MDAs per year in years 3 and 4. 

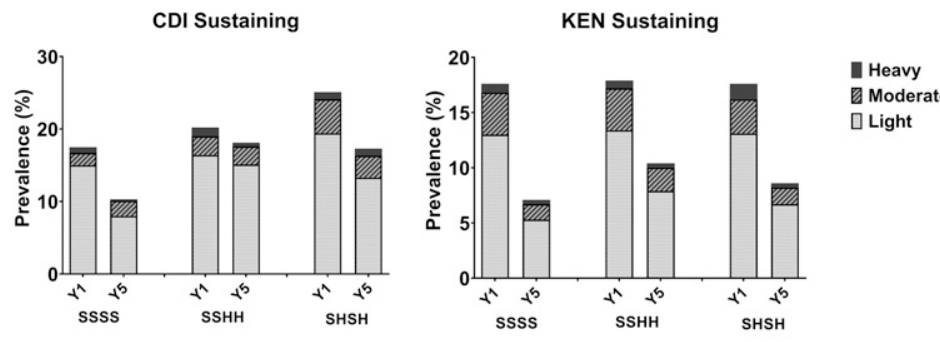

Moderat

Light

KEN Gaining

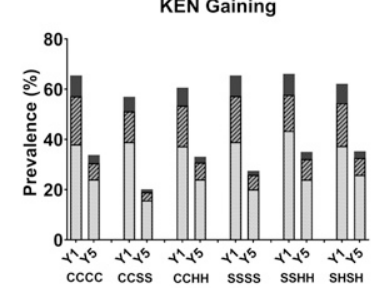

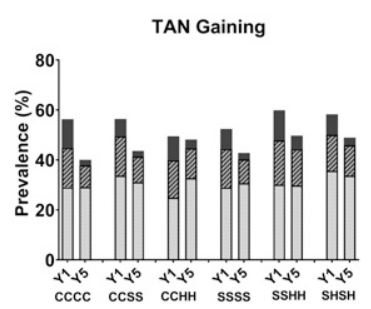

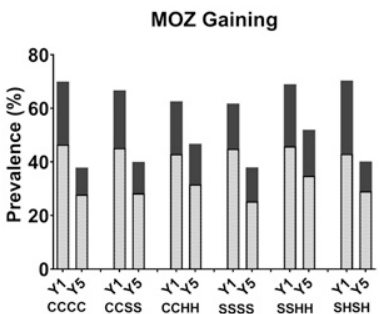

FIGURE 3. Overall prevalence, by infection categories of light, moderate, and heavy intensity categories at Year 1 (baseline; Y1) and Year 5 (study endpoint; Y5) in the Schistosomiasis Consortium for Operational Research and Evaluation gaining and sustaining control studies. The study arms indicated are detailed in Figure 1. $\mathrm{CDI}=$ Côte d'Ivoire; $\mathrm{C}=$ community-wide treatment; $\mathrm{H}=$ drug holiday-a year when no praziquantel mass drug administration was provided; KEN = Kenya; $\mathrm{MOZ}$ = Mozambique; $\mathrm{S}$ = school-based treatment; TAN = Tanzania.

infection (light, moderate, or heavy) ${ }^{9}$ are also indicated in Figure 3. Arms $3(\mathrm{CCHH})$ and $5(\mathrm{SSHH})$ in gaining control studies and Arm 2 (SSHH) in sustaining control studies involved skipping two consecutive years of praziquantel MDA as drug holidays. When compared with arms that had four annual treatments, those arms with alternate or consecutive holiday years had smaller reductions in prevalence in Côte d'Ivoire, Tanzania, Mozambique, and Kenya (see Supplemental Tables S1-S5). However, because of wide variability in individual community responses to their assigned MDA program, this apparent disadvantage of scheduled holidays was not statistically significant overall.
Prevalence of moderate and heavy infections declined among 9-12-year-old children in all study arms for all gaining and sustaining control studies. Figure 4 demonstrates the change in community-level mean infection intensity from Year 1 to Year 5 in the various studies.

Although there were initial reductions in response to MDA implementation, which accord with projections of pre-study deterministic modeling, ${ }^{29}$ the trajectories of aggregate prevalence and intensity impact tended to plateau after 2-3 years in most settings. ${ }^{30}$ We observed some larger-than-expected variations in the response of individual communities to MDA implementation, which we refer to as persistent hot spots. ${ }^{23,31}$
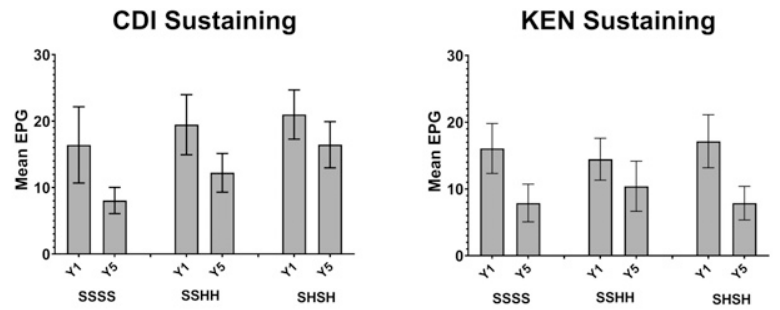

KEN Gaining

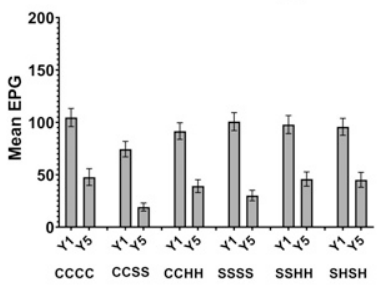

TAN Gaining

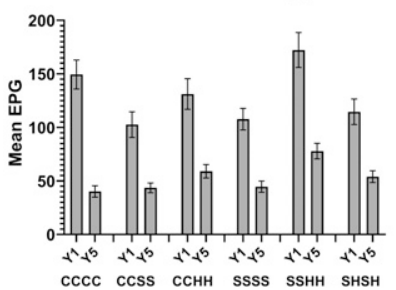

MOZ Gaining

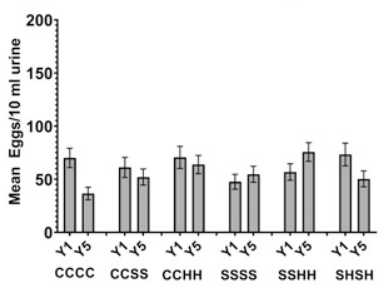

FIGURE 4. Mean community-level infection intensity in Schistosoma mansoni eggs per gram feces, or S. haematobium eggs per $10 \mathrm{~mL}$ urine at Year 1 (baseline; Y1) and Year 5 (study endpoint; Y5) in the Schistosomiasis Consortium for Operational Research and Evaluation gaining and sustaining control studies. The study arms indicated are detailed in Figure 1. $\mathrm{CDI}=\mathrm{Côte}$ d'lvoire; $\mathrm{C}=$ community-wide treatment; $\mathrm{H}=\mathrm{drug}$ holiday - a year when no praziquantel mass drug administration was provided; KEN = Kenya; MOZ = Mozambique; S = school-based treatment; TAN = Tanzania. 
CDI Sustaining Arm 1 (SSSS)

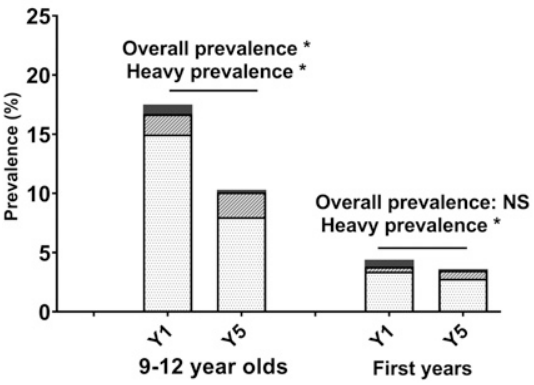

KEN Sustaining Arm 1 (SSSS)

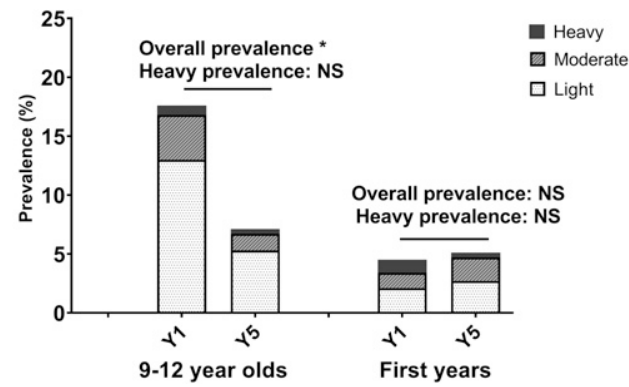

FIGURE 5. Impact on prevalence of four consecutive years of school-based treatment (SSSS) on 9-12-year-old and first-year schoolchildren in Schistosomiasis Consortium for Operational Research and Evaluation sustaining control studies. Baseline (Y1) and endpoint (Y5) prevalence of Schistosoma mansoni infection intensity categories are shown. Asterisks denote statistically significant differences between baseline and endpoint values at a $P<0.05$ level by $x^{2}$ testing. $\mathrm{CDI}=$ Côte d'Ivoire; $\mathrm{KEN}=$ Kenya; NS = not significant.

This phenomenon is discussed in greater detail in Article 3 of this supplement. ${ }^{32}$ The high degree of variance in response within each study arm meant that although there were, on average, large differences in prevalence and intensity impact (up to 40-60\%) between study arms, in our protocol's primary study outcomes analysis, which was specified before study completion (see Supplement File S2 for details), the betweenarm differences in prevalence and intensity effects were not statistically significant. Hence, there was no significant difference between the various schedules of coverage and delivery for MDA. Supplemental Tables S1-S5 in the Supplemental Material files provide the adjusted prevalence ratios and adjusted mean intensity ratios for the arm-to-arm comparisons in each of the SCORE gaining and sustaining studies. The very large intraclass correlations (40\%) observed in our studies mean that, if similar studies are performed in the future, it will be necessary to have a much higher number of villages per arm and fewer arms, or a completely different approach, such as a village-by-village evaluation of outcomes.

Additional analysis of the gaining and sustaining control studies. Our identification of persistent hot spots as a potential cause of limited overall response to MDA led us to perform an exploratory secondary analysis of communitylevel features that might explain the location-specific differences in outcomes. Baseline prevalence at the onset of intervention was different from community to community. However, this factor did not predict prevalence or intensity of infection in Year 5 (see Kittur et al., ${ }^{32}$ in this supplement, and

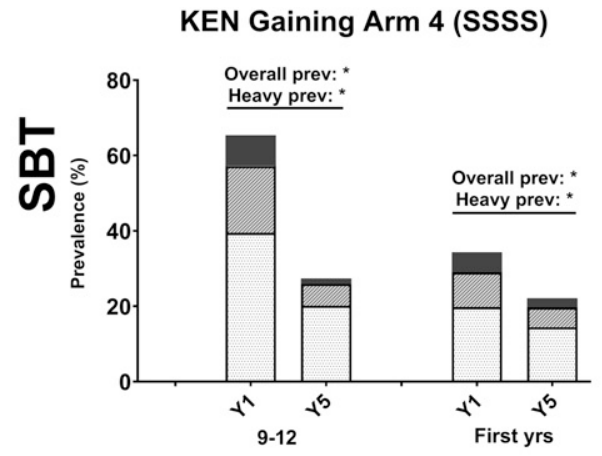

KEN Gaining Arm 1 (CCCC)

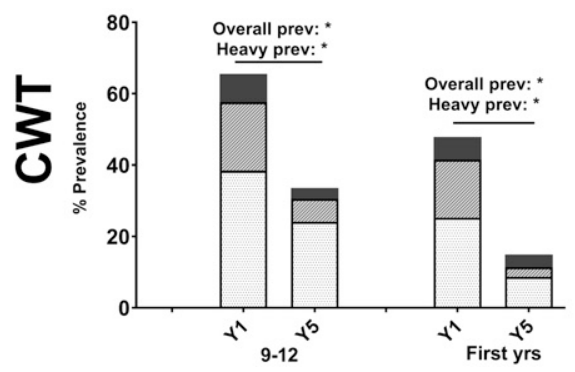

TAN Gaining Arm 4 (SSSS)

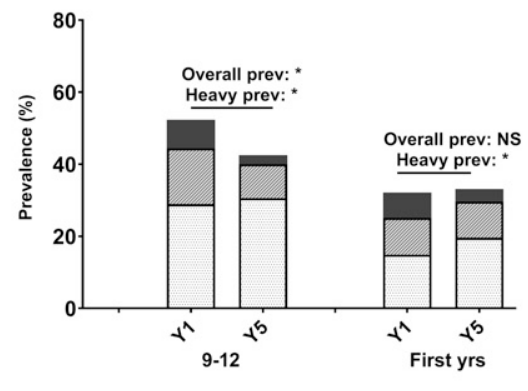

TAN Gaining Arm 1 (CCCC)

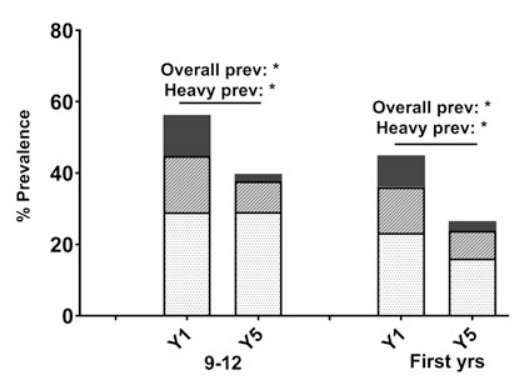

MOZ Gaining Arm 4 (SSSS)

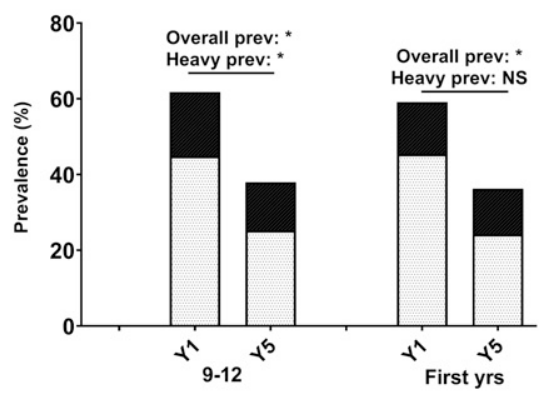

MOZ Gaining Arm 1 (CCCC)

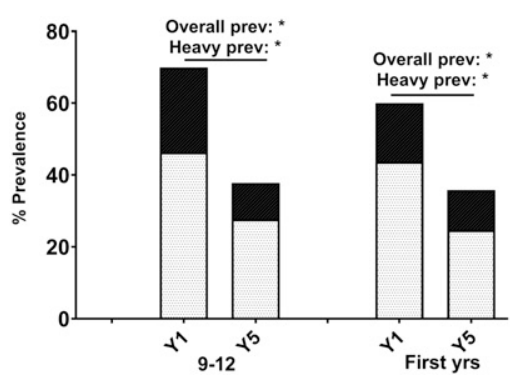

FIGURE 6. Impact on prevalence of four consecutive years of school-based treatment (SBT or SSSS) or four consecutive years of communitywide treatment (CWT or CCCC) on 9-12-year-old and first-year schoolchildren in Schistosomiasis Consortium for Operational Research and Evaluation gaining control studies. Baseline (Y1) and endpoint (Y5) prevalence of Schistosoma mansoni or Schistosoma haematobium infection intensity categories are shown. Asterisks denote statistically significant differences between baseline and endpoint values at a $P<0.05$ level by $X^{2}$ testing. $\mathrm{KEN}=$ Kenya; $\mathrm{MOZ}=$ Mozambique; NS = not significant; TAN = Tanzania. 
KEN Gaining Arm 4 (SSSS)

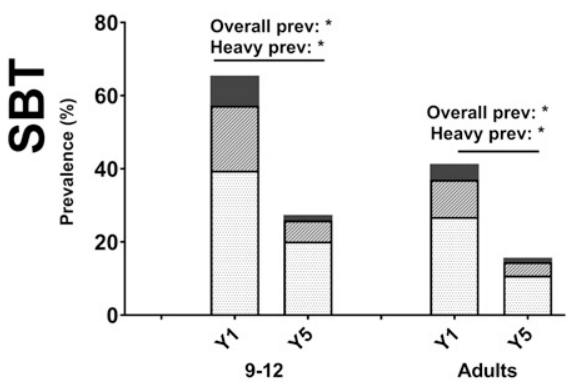

KEN Gaining Arm 1 (CCCC)

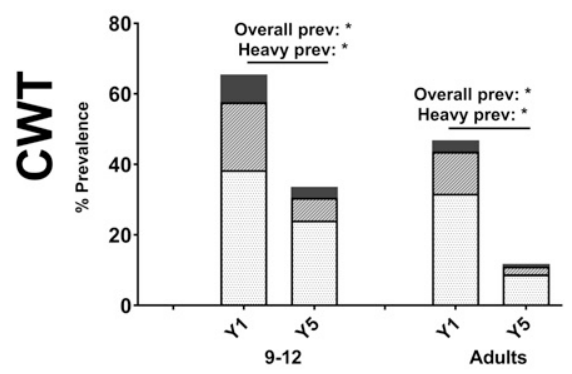

TAN Gaining Arm 4 (SSSS)

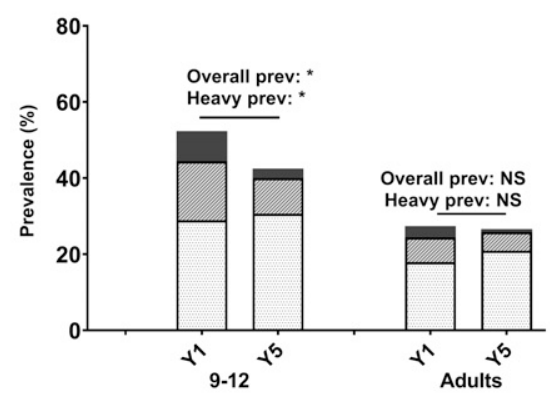

TAN Gaining Arm 1 (CCCC)

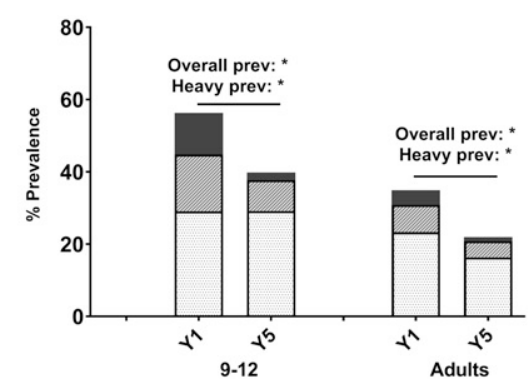

MOZ Gaining Arm 4 (SSSS)

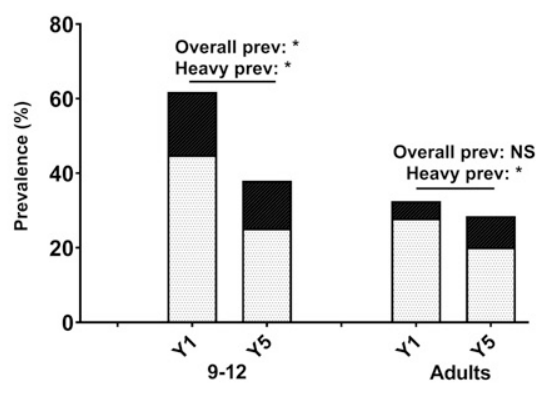

MOZ Gaining Arm 1 (CCCC)

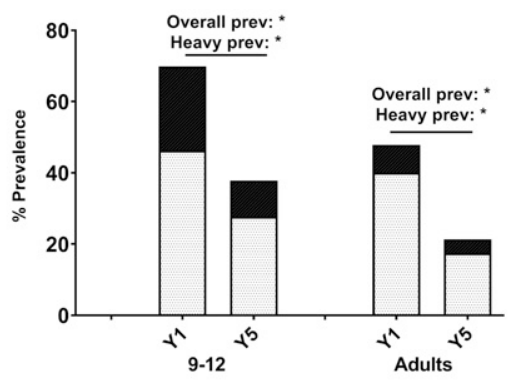

FIGURE 7. Impact of four consecutive years of school-based treatment (SBT or S; Arm 4) or four consecutive years of community-wide treatment (CWT or C; Arm 1) on 9-12-year-old children and adults in Schistosomiasis Consortium for Operational Research and Evaluation gaining control studies. Baseline (Y1) and endpoint (Y5) prevalence of Schistosoma mansoni infection intensity categories are shown. Asterisks denote statistically significant differences between baseline and endpoint values at a $P<0.05$ level by $x^{2}$ testing. KEN = Kenya; MOZ = Mozambique; NS = not significant; TAN = Tanzania.

Wiegand et al., ${ }^{23}$ ). Reported community-level MDA coverage was also not a good predictor of Year 5 outcomes. ${ }^{33}$ The use of open or unimproved water supplies, close proximity to open water sources, available sanitation (school or home latrines or toilets), and the practice of open defecation did not predict the odds of a community being a persistent hot spot (Musuva RM et al., personal communication). Geospatial clustering was observed for persistent hot spots in western Kenya, ${ }^{23}$ but not in Tanzania or Mozambique. Because the basic village inventory data collected during the SCORE treatment interventions were not revealing, SCORE has now sponsored follow-up "village factors" study in a matched subset of hot spot versus responder communities in Kenya and Tanzania to probe the community characteristics of hot spots in greater detail. Results of these surveys are pending, but we hope to identify the most influential local factors leading to poor response to MDA.

In its 2012-2020 Strategic Plan, the WHO recommended the following schistosomiasis targets: 1) morbidity control by reducing local prevalence of heavy Schistosoma infections to $<5 \%$ and 2) "elimination as a public health problem" (EPHP) by reducing the prevalence of heavy infections to $<1 \%$. Remarkably, as we took the SCORE MDA implementation to scale, we found that many (52-66\%) of the higher prevalence communities already met these criteria for morbidity control or for EPHP at implementation baseline, that is, before any MDA had been delivered. On the other hand, many persistent hot spot communities were unable to achieve the WHO targets for morbidity control despite 4 years of annual MDA with high levels of treatment coverage.
The use of local informants for collection of communitylevel data on environmental factors related to transmission, including issues such as water and sanitation, proved inconsistent. Similarly, we had concerns about reported treatment coverage data, which were not found to be helpful in explaining the overall results or the emergence of persistent hot spots. Details about these data are found in articles by Binder et al., ${ }^{34,35}$ in this supplement.

Impact of MDA on prevalence and intensity of Schistosoma infection in first-year students and adults. Earlier dynamic models had suggested that targeted mass treatment of SAC (who harbor most of the heavy-intensity infections in a typical endemic community) should reduce the force of local Schistosoma transmission in proportion to the reductions in overall egg output (i.e., reductions in community-level mean egg counts). ${ }^{36-38}$ Therefore, we looked for evidence of MDA-related changes in local force of transmission by monitoring the levels of infection among younger children (first-year school entrants aged 5-8 years) and among adults aged $20-55$ years in communities in gaining control studies, comparing baseline values to those observed in Year 5.

The impact of 4 years of SBT on the nontarget incoming firstyear students in sustaining control studies was minimal, with only a statistically significant decrease in heavy infections in Côte d'Ivoire and no impact on those in Kenya (Figure 5). By contrast, in areas of high prevalence that received 4 years of SBT (Figure 6, top row), a more consistent, although not uniform, impact was seen on the incoming first-year students, with a statistically significant decrease in either total prevalence and/ or prevalence of heavy infection. In parallel, in areas of high prevalence that received 4 years of CWT (Figure 6, bottom row), 


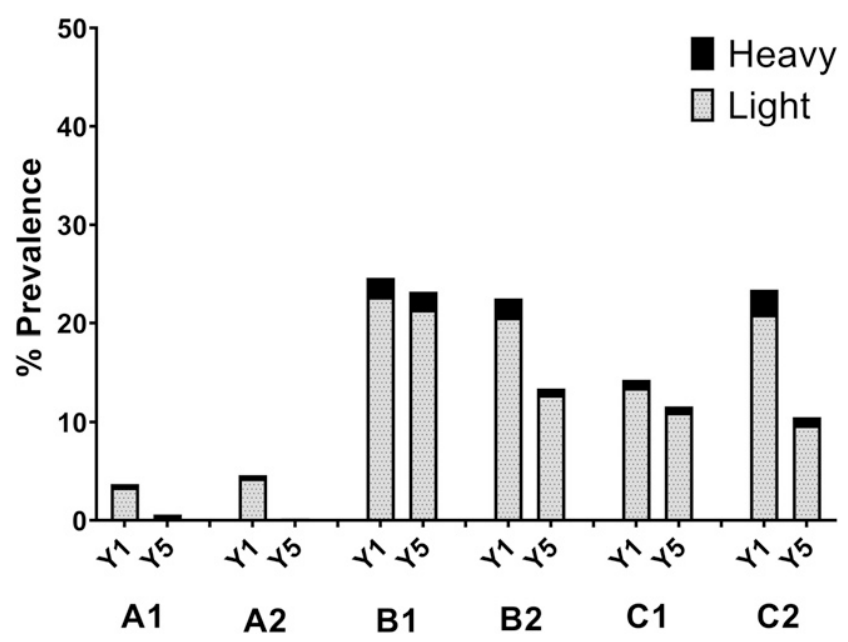

FIgURE 8. Outcomes of the Schistosomiasis Consortium for Operational Research and Evaluation Niger annual vs. biannual treatment trial. Shown are the starting (Y1) and ending (Y5) prevalences of heavy and light Schistosoma haematobium infections in communities that received treatment under the revised Niger study protocol. Studies A (in communities with microhematuria prevalence $5-20 \%$ in eligibility testing) and $B$ (communities with microhematuria prevalence $>20 \%$ in eligibility testing) began with annual or every-other-year school-based treatment, whereas Study C (communities with prevalence $>20 \%$ in eligibility testing) began with annual community-wide treatment during the first 2 years. Each study group was then randomized to receive annual or twice-yearly praziquantel treatments; creating groups $A 1$, $\mathrm{A} 2$, B1, B2, C1, and C2 (see Figure 2 for details). The difference in Y5 outcome was significant between groups B1 and B2 $(P=0.037)$. Differences in final prevalence outcomes for studies $A$ and $C$ were not statistically significant.

the prevalence and prevalence of heavy infections were significantly lower in the nontarget, younger age-group.

The gaining control studies also evaluated adults aged 20-55 years at the beginning and end of the 5-year study period. In arms that received 4 years of CWT, a modality that included adults in the MDA, both overall prevalence and prevalence of heavy infections were significantly reduced in all studies (Figure 7, bottom row). In arms that received 4 years of SBT only, where MDA did not include adults, the effects on adults varied by study (Figure 7, top row). In the Kenya gaining control study, the overall prevalence and prevalence of heavy infections were significantly reduced among adults in both SBT and CWT arms, indicating that 4 years of SBT was almost as effective as 4 years of CWT in reducing schistosome infection in the local adult population. By contrast, in Tanzania and Mozambique, SBT alone did not reduce schistosome infection in adults.

Niger study results. As shown in Figure 8, biannual SBT treatment was found to be significantly more effective for reducing active schistosome infection than annual SBT in higher prevalence areas (Arm B1 versus B2, defined in Figure 2). However, there was no significant effect of increased treatment frequency in areas with low starting prevalence (Arm A1 versus $A 2$ ), or in higher prevalence areas that received CWT instead of SBT (Arm C1 versus C2).

\section{SUMMARY AND PUBLIC HEALTH IMPLICATIONS}

To define the effectiveness and test the limitations of current WHO-recommended schistosomiasis control protocols, ${ }^{9,18}$ the
SCORE gaining and sustaining operational research projects performed randomized pragmatic trials that compared the impact of different schedules and coverage levels of praziquantel MDA. ${ }^{1}$ This field research was implemented on a much larger scale than any previous trials and under circumstances that would closely mirror implementation of MDA in national control programs. Although there were limitations to the study designs ${ }^{39}$ and performance, ${ }^{34}$ the results from all studies confirm the following:

1. Across all treatment schedules (study arms), MDA with praziquantel significantly reduced local Schistosoma infection prevalence and infection intensity among SAC.

2. Evidence from some study locations suggested that outcomes in locations receiving four annual rounds of MDA were better than those in communities that skipped years (i.e., only treated twice in 4 years). ${ }^{28}$ This effect was obscured by the wider-than-expected variation in communitylevel response to MDA, unmasking the persistent hot spot phenomenon. $^{23,33}$

3. Because individual community responses to standard MDA implementation were so highly variable, with many persistent hot spots, it was likely that some communities did not get the full benefits expected from MDA implementation. Therefore, for optimal MDA results, there is a need to identify persistent hot spots quickly, ${ }^{30}$ well before the 5-6 years currently recommended for treatment impact assessment in the current WHO strategies. ${ }^{40}$ For now, we would recommend at least annual SBT MDA to start, with earlier (Year 3) impact assessments, with ramp up of MDA performance, and nondrug interventions where needed. ${ }^{30}$

4. Although untreated adult populations have been identified as a potential source for persistence of transmission, ${ }^{41}$ the SCORE gaining control studies found that the results obtained by broadening population coverage (i.e., CWT versus SBT) were not better than those obtained with well-implemented SBT. There was evidence that in some moderate-to-high prevalence settings (Kenya, Mozambique, and Niger), SBT may have reduced infection levels among adults and new school entrants through indirect effects on local force of transmission (Phillips AE, et al., personal communication). ${ }^{24,28,30}$

5. None of the MDA schedules evaluated in the gaining and sustaining control studies led to elimination of infection, even in those communities that started at lower prevalence in the sustaining control studies. Therefore, it is likely that future programs aiming for elimination of transmission will need to add extra nondrug interventions (e.g., snail control, improvement in water, sanitation and hygiene, and behavior change interventions) to achieve that next stage of control.

These findings have been discussed in detail with the WHO NTD policy makers, BMGF, and other governmental and nongovernmental NTD control partners to aid in formulation of the next generation of schistosomiasis control and elimination guidelines.

Received November 6, 2019. Accepted for publication December 20, 2019.

Published online May 12, 2020.

Note: Supplemental appendix material and tables appear at www.ajtmh.org.

Financial support: These studies received financial support from University of Georgia Research Foundation, Inc., which was funded by 
the Bill \& Melinda Gates Foundation for the SCORE project. In addition, a significant amount of the praziquantel provided as treatment during these studies was donated through the WHO, the United States Agency for International Development (USAID), RTI International, World Vision, and the Schistosomiasis Control Initiative (SCl). The funders had no role in the study design, data collection and analysis, decision to publish, or preparation of the manuscript.

Trials registration: The studies discussed in this article are registered at International Standard Randomised Controlled Trial registry (http:// www.isrctn.com/). Identifiers: ISRCTN99401114 (Côte d'Ivoire Sustaining Control Study (Sm1)), ISRCTN14849830 (Kenya Sustaining Control Study (Sm1)), ISRCTN16755535 (Kenya Gaining Control Study (Sm2)), ISRCTN14117624 (Mozambique Gaining Control Study (Sh2)), ISRCTN95819193 (Tanzania Gaining Control Study (Sm2)), and ISRCTN32045736 (Niger annual versus biannual treatment study).

Data availability: Data collected in SCORE studies will be housed and made accessible to the global research community through Clinical Epidemiology Database Resources, ClinEpiDB (https://clinepidb.org/ ce/app/) at the University of Pennsylvania. Data will be available as anonymized individual participant data with an accompanying data dictionary in early 2020 .

Disclaimer: The findings and conclusions in this report are those of the authors and do not necessarily represent the views of the U.S. Centers for Disease Control and Prevention or of the World Health Organization.

Authors' addresses: Charles H. King, Center for Global Health and Diseases, CWRU School of Medicine, Cleveland, OH, E-mail: chk@ cwru.edu. Nupur Kittur, Sue Binder, Carl H. Campbell Jr., and Daniel G. Colley, Schistosomiasis Consortium for Operational Research and Evaluation, Center for Tropical and Emerging Global Diseases, University of Georgia, Athens, GA, E-mails: nkittur@uga.edu, suebinder1@ gmail.com, ccamp@uga.edu, and dcolley@uga.edu. Eliézer K. N'Goran, Unité de Formation et de Recherche Biosciences, Université Félix Houphouët-Boigny, Abidjan, Côte d'Ivoire, E-mail: eliezerngoran@ yahoo.fr. Aboulaye Meite, Programme National de Lutte Contre les Maladies Tropicales Négligées à Chimiothérapie Préventive (PNLMTN-CP), Abidjan, Côte d'Ivoire, E-mail: aboulaye_meite77@yahoo.fr. Jürg Utzinger, Swiss Tropical and Public Health Institute, Basel, Switzerland, E-mail: juerg.utzinger@swisstph.ch. Annette Olsen, Section for Parasitology and Aquatic Pathobiology, Faculty of Health and Medical Sciences, University of Copenhagen, Copenhagen, Denmark, E-mail: aol@ sund.ku.dk. Pascal Magnussen, Centre for Medical Parasitology, Faculty of Health and Medical Sciences, University of Copenhagen, Copenhagen, Denmark, E-mail: pma@sund.ku.dk. Safari Kinung'hi, National Institute for Medical Research, Mwanza, Tanzania, E-mail: kinunghi_csm@ hotmail.com. Alan Fenwick and Neerav Dhanani, Department of Infectious Disease Epidemiology, Schistosomiasis Control Initiative, Imperial College, London, United Kingdom, E-mails: a.fenwick@ imperial.ac.uk and n.dhanani@schisto.org.uk. Anna E. Phillips, Department of Infectious Disease Epidemiology, London Centre for Neglected Tropical Disease Research (LCNTDR), London, United Kingdom, E-mail: a.phillips05@imperial.ac.uk. Pedro H. Gazzinelli-Guimaraes, NIAID, National Institutes of Health, Bethesda, MD, E-mail: pedro.gazzinelliguimaraes@nih.gov. Josefo Ferro, Faculty of Health Sciences, Universidade Católica de Moçambique (UCM), Beira, Mozambique, E-mail: josefoferro@yahoo.com.br. Diana M. S. Karanja, Centre for Global Health Research, Kenya Medical Research Institute, Kisumu, Kenya, E-mail: diana@cohesu.org. Pauline N. M. Mwinzi, WHO/AFRO Regional Office Neglected Tropical Diseases, Brazzaville, Republic of the Congo, E-mail: mwinzip@who.int. Susan P. Montgomery, Ryan E. Wiegand, and William Evan Secor, Division of Parasitic Diseases and Malaria, Parasitic Diseases Branch, Centers for Disease Control and Prevention, Atlanta, GA, E-mails: zqu6@cdc.gov, fwk2@cdc.gov, and was4@ cdc.gov. Amina A. Hamidou, Réseau International Schistosomoses, Environnement, Aménagement et Lutte (RISEAL-Niger), Niamey, Niger, E-mail: aminamadou@yahoo.fr. Amadou Garba, Department of Control of Neglected Tropical Diseases, Preventive Chemotherapy and Transmission Control Unit, World Health Organization, Geneva, Switzerland, E-mail: garbadjirmaya@who.int.

This is an open-access article distributed under the terms of the Creative Commons Attribution (CC-BY) License, which permits unrestricted use, distribution, and reproduction in any medium, provided the original author and source are credited.

\section{REFERENCES}

1. Ezeamama AE et al., 2016. Gaining and sustaining schistosomiasis control: study protocol and baseline data prior to different treatment strategies in five African countries. BMC Infect Dis 16: 229.

2. Burnim M, Ivy JA, King $\mathrm{CH}, 2017$. Systematic review of community-based, school-based, and combined delivery modes for reaching school-aged children in mass drug administration programs for schistosomiasis. PLoS Negl Trop Dis 11: e0006043.

3. Leslie J, Garba A, Oliva EB, Barkire A, Tinni AA, Djibo A, Mounkaila I, Fenwick A, 2011. Schistosomiais and soil-transmitted helminth control in Niger: cost effectiveness of school based and community distributed mass drug administration. PLoS Negl Trop Dis 5: e1326.

4. Homeida MM, Eltoum IA, Ali MM, Suliaman SM, Elobied EA, Mansour M, Saad AM, Bennett JL, 1996. The effectiveness of annual versus biennial mass chemotherapy in reducing morbidity due to schistosomiasis: a prospective study in GeziraManagil, Sudan. Am J Trop Med Hyg 54: 140-145.

5. Garba A, Campagne G, Tassie JM, Barkire A, Vera C, Sellin B, Chippaux JP, 2004. Long-term impact of a mass treatment by praziquantel on morbidity due to Schistosoma haematobium in two hyperendemic villages of Niger [article in French]. Bull Soc Pathol Exot 97: 7-11.

6. Li YS, Sleigh AC, Ross AG, Li Y, Williams GM, Tanner M, McManus DP, 2000. Two-year impact of praziquantel treatment for Schistosoma japonicum infection in China: re-infection, subclinical disease and fibrosis marker measurements. Trans $R$ Soc Trop Med Hyg 94: 191-197.

7. Magnussen $P$, Muchiri E, Mungai $P$, Ndzovu M, Ouma J, Tosha S, 1997. A school-based approach to the control of urinary schistosomiasis and intestinal helminth infections in children in Matuga, Kenya: impact of a two-year chemotherapy programme on prevalence and intensity of infections. Trop Med Int Health 2: 825-831.

8. Hatz CF, Vennervald BJ, Nkulila T, Vounatsou P, Kombe $Y$, Mayombana C, Mshinda H, Tanner M, 1998. Evolution of Schistosoma haematobium-related pathology over 24 months after treatment with praziquantel among school children in southeastern Tanzania. Am J Trop Med Hyg 59: 775-781.

9. WHO, 2006. Preventive Chemotherapy in Human Helminthiasis: Coordinated Use of Anthelminthic Drugs in Control Interventions: A Manual for Health Professionals and Programme Managers. Geneva, Switzerland: World Health Organization.

10. Shen $Y$ et al., 2017. Protocol and baseline data for a multi-year cohort study of the effects of different mass drug treatment approaches on functional morbidities from schistosomiasis in four African countries. BMC Infect Dis 17: 652.

11. Campbell CH et al., 2020. SCORE operational research on moving toward interruption of Schistosomiasis transmission. Am J Trop Med Hyg 103 (Suppl 1): 58-65.

12. Tian-Bi YT et al., 2018. Interrupting seasonal transmission of Schistosoma haematobium and control of soil-transmitted helminthiasis in northern and central Cote d'Ivoire: a SCORE study protocol. BMC Public Health 18: 186.

13. Katz N, Chaves A, Pellegrino J, 1972. A simple device for quantitative stool thick-smear technique in schistosomiasis mansoni. Rev Inst Med Trop Sao Paulo 14: 397-400.

14. King CH, Bertsch D, 2013. Meta-analysis of urine heme dipstick diagnosis of Schistosoma haematobium infection, including low-prevalence and previously-treated populations. PLoS Negl Trop Dis 7: e2431.

15. Lengeler $C$, Mshinda $H$, Morona $D$, deSavigny $D$, 1993. Urinary schistosomiasis: testing with urine filtration and reagent sticks for haematuria provides a comparable prevalence estimate. Acta Trop 53: 39-50.

16. King $\mathrm{CH}$ et al., 1990. Chemotherapy-based control of Schistosomiasis haematobia. II. Metrifonate vs. praziquantel in control of infection-associated morbidity. Am J Trop Med Hyg 42: 587-595. 
17. Knopp S, Ame SM, Hattendorf J, Ali SM, Khamis IS, Bakar F, Khamis MA, Person B, Kabole F, Rollinson D, 2018. Urogenital schistosomiasis elimination in Zanzibar: accuracy of urine filtration and haematuria reagent strips for diagnosing light intensity Schistosoma haematobium infections. Parasit Vectors 11: 552.

18. WHO, 2002. Prevention and control of schistosomiasis and soiltransmitted helminthiasis: report of a WHO expert committee. Technical Report Series 912. Geneva, Switzerland: World Health Organization, 1-57.

19. King $\mathrm{CH}$ et al., 2020. SCORE studies on the impact of drug treatment on morbidity due to Schistosoma mansoni and Schistosoma haematobium infection Am J Trop Med Hyg 103 (Suppl 1): 30-35.

20. Allan F et al., 2020. Snail-related contributions from the Schistosomiasis Consortium for Operational Research and Evaluation program including xenomonitoring, focal mollusciciding, biological control, and modeling. Am J Trop Med Hyg 103 (Suppl 1): 66-79.

21. Webster JP, Neves MI, Webster BL, Pennance T, Rabone M, Gouvras AV, Allan F, Walker M, Rollinson D, 2020. Parasite population genetic contributions to the Schistosomiasis Consortium for Operational Research and Evaluation within SubSaharan Africa. Am J Trop Med Hyg 103 (Suppl 1): 80-91.

22. Karanja DMS, Awino EK, Wiegand RE, Okoth E, Abudho BO, Mwinzi PNM, Montgomery SP, Secor WE, 2017. Cluster randomized trial comparing school-based mass drug administration schedules in areas of western Kenya with moderate initial prevalence of Schistosoma mansoni infections. PLoS Negl Trop Dis 11: e0006033.

23. Wiegand RE et al., 2017. A persistent hotspot of Schistosoma mansoni infection in a five-year randomized trial of praziquantel preventative chemotherapy strategies. J Infect Dis 216: $1425-1433$.

24. Phillips AE, Gazzinelli-Guimaraes $\mathrm{PH}$, Aurelio HO, Ferro J, Nala R, Clements M, King $\mathrm{CH}$, Fenwick A, Fleming FM, Dhanani N, 2017. Assessing the benefits of five years of different approaches to treatment of urogenital schistosomiasis: a SCORE project in northern Mozambique. PLoS Negl Trop Dis 11: e0006061.

25. Assare RK et al., 2014. Sustaining control of Schistosomiasis mansoni in moderate endemicity areas in western Cote d'Ivoire: a SCORE study protocol. BMC Public Health 14: 1290.

26. Olsen A, Kinung'hi S, Magnussen P, 2018. Comparison of the impact of different mass drug administration strategies on infection with Schistosoma mansoni in Mwanza region, Tanzania-a cluster-randomized controlled trial. Am J Trop Med Hyg 99: 1573-1579.

27. Assare RK et al., 2016. Sustaining control of schistosomiasis mansoni in western Cote d'Ivoire: results from a SCORE study, one year after initial praziquantel administration. PLOS Negl Trop Dis 10: e0004329.

28. Secor WE, Wiegand RE, Montgomery SP, Karanja DM, Odiere MR, 2020. Comparison of school-based and community-wide mass drug administration for schistosomiasis control in an area of western Kenya with high initial Schistosoma mansoni infection prevalence: a cluster randomized trial. Am J Trop Med Hyg 102: 318-327.
29. Wang X, Gurarie D, Mungai PL, Muchiri EM, Kitron U, King CH, 2012. Projecting the long-term impact of school- or community-based mass-treatment interventions for control of Schistosoma infection. PLoS Negl Trop Dis 6: e1903.

30. Li E, Gurarie D, Lo NC, Zhu X, King CH, 2019. Improving public health control of schistosomiasis with a modified WHO strategy: a model-based comparison study. Lancet Glob Health 7: e1414-e1422.

31. Kittur N, Binder S, Campbell CH, King CH, Kinung'hi S, Olsen A, Magnussen P, Colley DG, 2017. Defining persistent hotspots: areas that fail to decrease meaningfully in prevalence after multiple years of mass drug administration with praziquantel for control of schistosomiasis. Am J Trop Med Hyg 97: 1810-1817.

32. Kittur $\mathrm{N}$ et al., 2020. Discovering, defining, and summarizing persistent hotspots in SCORE studies. Am J Trop Med Hyg 103 (Suppl 1): 24-29.

33. Kittur $\mathrm{N}$ et al., 2019. Persistent hotspots in SCORE studies for gaining and sustaining control of schistosomiasis after four years of mass drug administration of praziquantel. $A m \mathrm{~J}$ Trop Med Hyg 101: 617-627.

34. Binder S, Campbell CH, Andros T, Castleman JD, Kittur N, King $\mathrm{CH}$, Colley DG, 2020. The Schistosomiasis Consortium for Operational Research and Evaluation 2008-2020: approaches, experiences, lessons, and recommendations. Am J Trop Med Hyg 103 (Suppl 1): 114-124.

35. Binder $S$ et al., 2020. Lessons learned in conducting mass drug administration for Schistosomiasis control and measuring coverage in an operational research setting. Am J Trop Med Hyg 103 (Suppl 1): 105-113.

36. Anderson RM, Turner HC, Farrell SH, Yang J, Truscott JE, 2015. What is required in terms of mass drug administration to interrupt the transmission of schistosome parasites in regions of endemic infection? Parasit Vectors 8: 553.

37. Warren KS, Bundy D, Anderson RM, Davis A, Henderson DA, Jamison DT, Prescott N, Senft A, 1990. Helminth infections. Jamison DT, Mosley WH, eds. Evolving Health Sector Priorities in Developing Countries. Washington, DC: The World Bank.

38. French MD et al., 2015. Estimation of changes in the force of infection for intestinal and urogenital schistosomiasis in countries with Schistosomiasis Control Initiative-assisted programmes. Parasit Vectors 8: 558.

39. King $\mathrm{CH}$, Kittur N, Wiegand RE, Shen Y, Ge Y, Whalen CC, Campbell C, Hattendorf J, Binder S, 2020. Challenges in protocol development and interpretation of the Schistosomiasis Consortium for Operational Research and Evaluation intervention studies. Am J Trop Med Hyg 103 (Suppl 1): $36-41$.

40. WHO, 2013. Schistosomiasis: Progress Report 2001-2011, Strategic Plan 2012-2020. Geneva, Switzerland: World Health Organization, 81.

41. Toor J, Turner HC, Truscott JE, Werkman M, Phillips AE, Alsallaq R, Medley GF, King CH, Anderson RM, 2018. The design of schistosomiasis monitoring and evaluation programmes: the importance of collecting adult data to inform treatment strategies for Schistosoma mansoni. PLoS Negl Trop Dis 12: e0006717. 